\title{
Mechanical influences on bone development in children
}

\author{
E Schoenau and O Fricke \\ Children's Hospital, University of Cologne, Kerpener Strasse 62, D-50924 Cologne, Germany \\ (Correspondence should be addressed to E Schoenau; Email: eckhard.schoenau@uk-koeln.de)
}

\begin{abstract}
This review focuses on methodological concepts in the evaluation of skeletal muscle function and on adaptation. It is now thought that the critical property of bone is strength rather than weight, and that control of bone strength is mainly exercised through the effect of the mechanical loads brought to bear on bone. Muscle contraction places the greatest physiological load on bone, and so the stability of bone must be adapted to muscle strength (the functional muscle-bone unit). The described suggestions and recommendations outline a new concept: bone mass and strength should not be related to age. There is now more and more evidence that bone mass and strength should be related to muscle function. Thus analyzed, there is no such entity as 'peak bone mass'. Many studies are presently under way to evaluate whether these novel approaches increase the sensitivity and specificity of fracture prediction in an individual. Furthermore, the focus of many bone researchers is shifting away from bone mass to bone geometry or bone strength and their relationship with the driving muscle system.
\end{abstract}

European Journal of Endocrinology 159 S27-S31

\section{Introduction}

Each generation challenges the health care system with new demands as the prevalence of medical problems requiring preventive and curative approaches is constantly changing. In western industrialized countries, most individuals have replaced previously common motor activities of daily life with the technical assistance provided by a mechanized and computerized world. Because the musculoskeletal system is adapting to biomechanical challenges and environmental conditions, the body composition of the average child and adolescent has dramatically changed in western societies $(1,2)$. Insulin resistance, obesity, osteopenia, and sarcopenia are now typical challenges facing health care providers dealing with disease prevention and health education. Until a few decades ago, pediatric health care in western societies focused on the prevention of rickets and malnutrition due to low caloric intake. Nowadays, the urgent issues are an atrophic musculoskeletal system caused by low motor activity and malnutrition due to high caloric intake. In addition, the improved care of chronically ill children introduced the issue of secondary musculoskeletal diseases. Therefore, knowledge and know-how on the evaluation of muscle-skeletal interactions will become more of an issue in pediatrics. Knowledge on the

This paper was presented at the 5th Ferring International Paediatric Endocrinology Symposium, Baveno, Italy (2008). Ferring Pharmaceuticals has supported the publication of these proceedings. development of the musculoskeletal systems and the assessment of this process will become important topics for those who are responsible for improving the level of medical care in our societies. This review discusses the mechanical properties of skeletal development.

\section{The peak bone mass concept}

The central tenet of this concept is that attainment of peak bone mass during childhood and adolescence will prevent fractures in later life, and is based on the observation that areal bone density reaches a peak at around 20 years of age and then decreases. Two possibilities may account for the occurrence of fractures in later life: either such individuals have lost more bone than those without fractures, or their peak bone mass was lower in young adulthood and thereafter they lost bone mass at the same rate and for the same length of time as those without fractures (3).

In this context, it is important to distinguish between bone mass and density: bone mass equals the weight of the bone, which depends on bone size, while the physical bone density represents the bone mineral content (BMC) relative to the outer bone volume and is independent of size. A normal small bone will have a lower areal density than a larger one, even if their physical densities are identical, and so a presumed bone mass deficit may disappear when the results are corrected for body height or bone size (3).

Moreover, heavy bones will increase energy expenditure and decrease running speed and are therefore 
unlikely to have conferred an evolutionary advantage (4). Furthermore, serum calcium remains stable even in the presence of severe osteopenia, and hence maximization of calcium stores through increased skeletal weight is unlikely to be functionally advantageous (5).

Therefore, the most critical property of bone for survival is now thought to be its strength rather than its weight. Since fracture of a bone would have resulted in death in the wild, it is logical that humans would develop mechanisms during the evolutionary process that would encourage bone development to produce bones of optimal strength (4).

\section{Harold Frost's mechanostat hypothesis}

The combination of factors that make healthy loadbearing bones satisfy the needs of all amphibians, birds, mammals, and reptiles of any size, age, and sex was named the mechanostat. It combines the modeling and remodeling mechanisms, their thresholds, the marrow mediator mechanisms, the signaling mechanisms that connect them, and perhaps other things. For mechanical reasons, the resulting negative feedback system determines whether, when and where bones need more strength, or when bone is not needed. Various nonmechanical factors, including hormones and other humoral agents, might modulate ('help or hinder') the mechanostat's effects on bone strength. The mechanostat could be compared with the combination of a car's steering, brakes, and accelerator. Osteoblasts and osteoclasts could be analogous to the car's wheels, and mechanical usage its driver (Fig. 1) (6-8). The development of muscle and bone during growth is influenced by forces associated with gravity and physical activity $(7,9$, $10)$. It is the muscle forces that create the peak forces acting on bone. Thus, growth in the presence of unloading results in both a muscle that lacks functional capacity, and a bone that lacks the specific shape that is unique for its function (11). This intrinsic relationship between muscle and bone is described by the mechanostat theory which postulates that increasing maximal muscle force during growth or in response to increased loading will affect bone mass, size, and strength. Unloading (disuse or immobilization) will lead to reduced muscle development (and muscle force) and have a negative effect on the mass, size, and strength of bone. The proper functioning of the mechanostat depends on the normal state of all its cells (osteocytes, osteoblasts, and osteoclasts), the customary mechanical usage of the skeleton, and the endocrinemetabolic environment (12). The fine-tuning of the mechanostat is achieved by physiological set points that act as thresholds for the initiation or inhibition of bone modeling and remodeling. Mechanostat set points are genetically determined and are regulated by the endocrine environment. For instance, it is proposed that reduced estrogen concentrations increase the set points for bone modeling and remodeling. The endocrine environment affects the mechanostat sensitivity with which bone adapts its mass, geometry, or structural properties to bone deformations caused by loading (12). Exercise and nutrition are key environmental factors known to affect muscle and bone development. Exercise acts directly through muscle action and indirectly through endocrine regulation; during growth, exercise is thought to influence bone modeling and thus bone geometry.

\section{Development of the 'functional muscle- bone unit'}

Figure 2 shows the relationships between age and trabecular density; between BMC and bone strength index (BSI); and between muscle area and trabecular

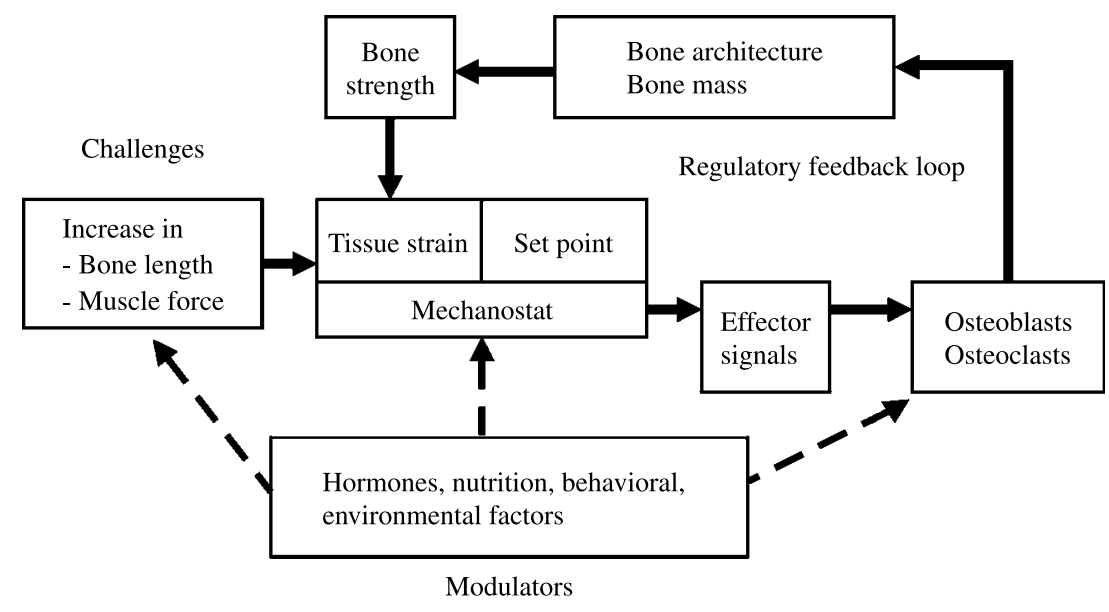

Figure $1 \mathrm{~A}$ functional model of bone development based on the mechanostat theory. The central piece of bone regulation is the feedback loop between bone deformation (tissue strain) and bone strength. During growth, this homeostatic system is continually forced to adapt to external challenges. The factors shown below modulate various aspects of the central regulatory system. From Rauch \& Schoenau (5), with permission. 

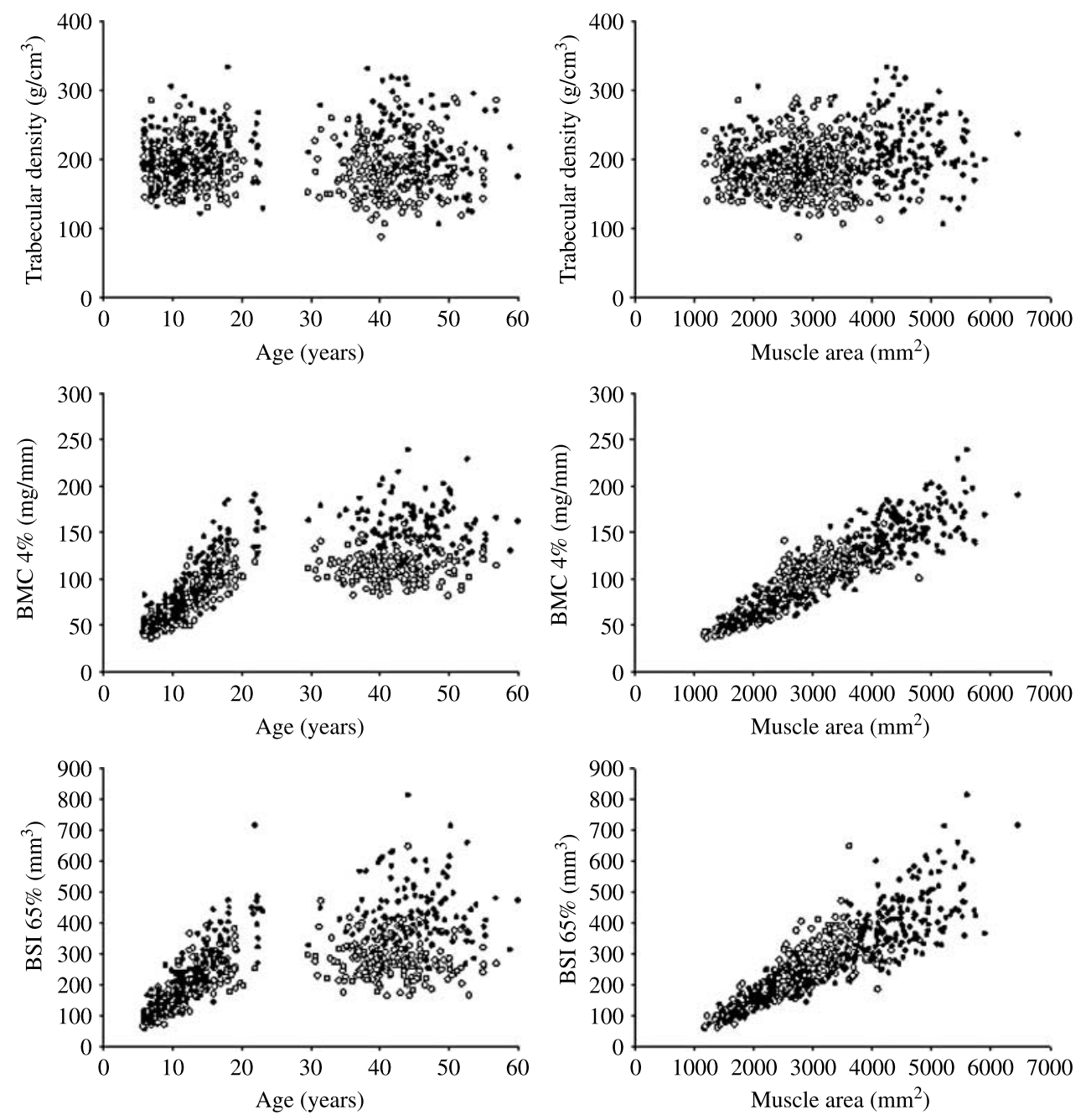

Figure 2 (a) Trabecular density, (b) bone mineral content (BMC), and (c) bone strength index (BSI) in relation to age and muscle area in a healthy reference population at the distal radius. From Schoenau (16), with permission.

density, and BMC and BSI in a healthy reference population. Anthropometric data and results obtained with peripheral quantitative computed tomography (pQCT) in these individuals have been reported previously (13-15). Trabecular density as an index of tissue density is dependent on neither age nor muscle development, whereas BMC and BSI appear to be dependent on age during childhood only. By contrast, BMC and BSI show a strong linear correlation with muscle development in childhood and adulthood. These data show that bone density is more or less 'constant', and that BMC and BSI are a function of muscle development.

Based on these considerations, some years ago we recommended relating analyzed bone data with surrogates of muscle development. Instead of using agerelated reference data, analysis of the so-called 'functional muscle-bone unit' (Fig. 3) should improve understanding of the physiology and pathophysiology of bone development.

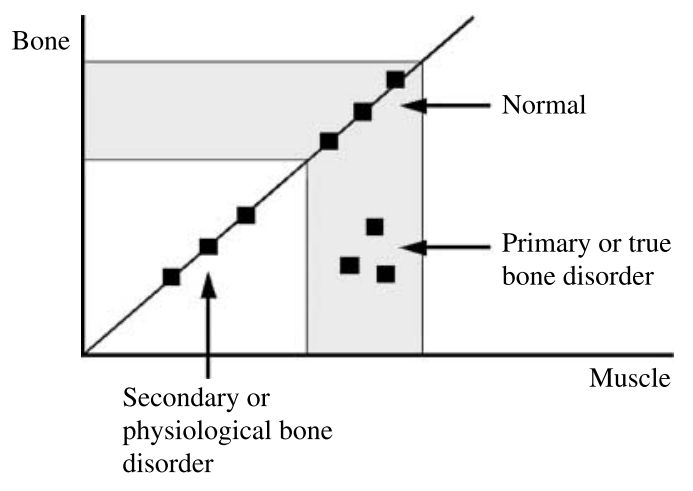

Figure 3 The 'functional muscle-bone unit'. In the cases of 'primary bone disease', the bone structure/mass is not adapted to muscle development. In the cases of 'secondary bone disease', there is disturbed muscle development, but a normal adapted skeleton. From Schoenau (16), with permission. 


\section{A new algorithm in diagnostics and treatment of metabolic bone diseases}

We proposed a two-stepped diagnostic algorithm to characterize metabolic bone diseases in children and adolescents (Fig. 4) (16-18). This algorithm includes two important aspects of the skeletal development in children and adolescents - the relationship of bone mass with body height and muscle force. In the first step, muscle mass is referred to height. In the second step, the ratio of bone mass to muscle force (expressed by crosssectional muscle area or maximal force) indicates whether the individual is characterized by intact bone, or a primary or a secondary bone disease.

Thereby, bone parameters are measured by densitometric methods under consideration that the volumetric approach is preferred in children and adolescents. Suitable muscular parameters are maximal forces, e.g. maximal isometric grip force (MIGF), or a related parameter, e.g. the cross-sectional muscle area. A strong functional relationship between skeletal element and muscle are essential to obtain valid results. Based on our data, we recommend the cross-sectional muscle area at the $65 \%$ side in relation to BMC of the radius at the $4 \%$ side in pQCT analysis (19). Alternatively, crosssectional muscle area can be replaced by the functional parameter MIGF (20). This new algorithm has a tremendous effect on our understanding to treat bone diseases (21). Is it reasonable to treat an individual with vitamin $\mathrm{D}$ when the algorithm indicates a secondary bone disease? Are estrogens useful in an isolated therapeutic approach to decrease bone resorption in females whose problem is a mixed - primary and secondary (sarcopenia) - bone disease? The logical resume of this new algorithm is to classify therapeutic strategies due to this diagnostic concept. Primary bone diseases can be divided into a structural and functional pathology. Exemplarily, individuals affected with osteogenesis imperfecta are characterized by a structural deficit of the collagen molecule, and therefore these individuals profit from a therapy inhibiting bone

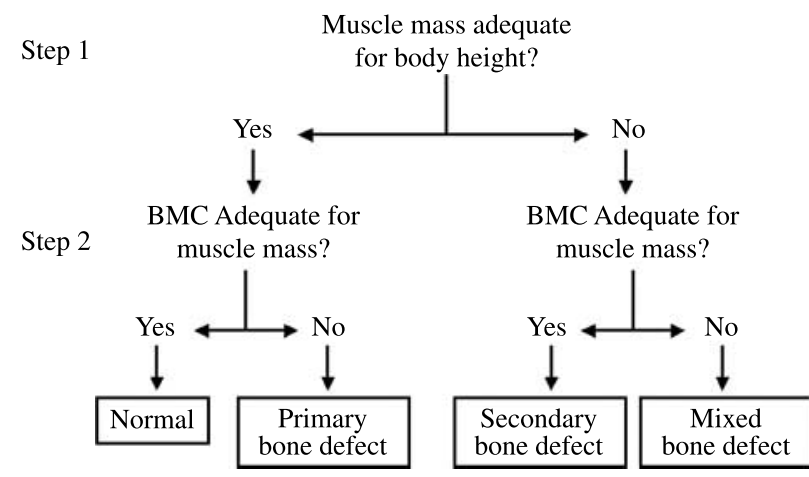

Figure 4 Proposed diagnostic two-step algorithm. Reproduced from Schoenau et al. (17) with permission of the American Society for Bone and Mineral Research. resorption (e.g. bisphosphonates) (22). Also, these individuals are characterized by secondary functional problems, because frequent fractures lead to immobility starting a circulus vitiosus: less muscle force induces more bone resorption followed by more fractures, decreased physical activity, and further decrease in muscular forces finally. Therefore, primary bone diseases are often combined with a secondary functional deficit in the 'functional muscle-bone unit' and profit from a specific physiotherapy. By contrast, secondary bone diseases might occur without any primary component as we exemplarily described in patients affected with cystic fibrosis (Fig. 5 (23)).

\section{Designing smart medicine under reflection of the mechanostat paradigm}

Finally, reasoning about the roots of hormonal action and the biological function of the skeletal system let us undertake a journey from a static concept to a dynamic understanding of bone diseases in childhood and adolescence. Harold Frost and his straightway back to Lucius Wolff's principle that tissue morphology and function are undividable for a correct understanding of biological processes in nature (24). When bone diseases are closely associated with muscular function - because mechanical stimuli are essential elements in the feedback loop regulating bone development - the pediatric osteologist has to be an expert in the diagnostics of muscular function. Actually, we are standing at the beginning of understanding key principles in the clinical analysis of the muscular function by the use of physical parameters as velocity, acceleration, force, energy, and power. Recently, mechanographic analyses of jumping

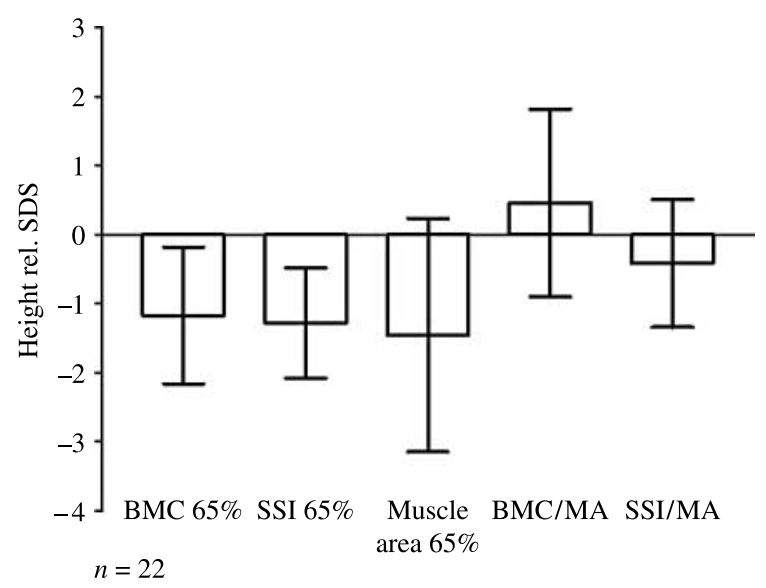

Figure 5 Development of muscle and bone in age-related SDS in individuals with cystic fibrosis. Bone mineral count (BMC) and cross-sectional muscle area (MA) are below the reference value. By contrast, the ratio of the BMC and the strength strain index (SSI) to the cross-sectional muscle area is not below the reference value, indicating a secondary osteopenia. From Fricke \& Schoenau (23), with permission. 
and chair-rising were introduced in the diagnostic repertoire of the pediatric osteologist (25). Actually, we have many open questions in pediatric biomechanics. Are maximal forces the essential parameter modeling bone and what is the optimal frequency at which these forces should be applied to support the increase in bone mass? In the future, a precise answer to these open questions will be necessary for a sufficient understanding of the physiology in bone diseases.

\section{Disclosure}

This paper forms part of a European Journal of Endocrinology supplement, supported by Ferring Pharmaceuticals. The authors disclose: no potential conflicting relationship with Ferring. This article was subject to rigorous peer review before acceptance and publication.

\section{References}

1 Strock GA, Cottrell ER, Abang AE, Buschbacher RM \& Hanson TS Childhood obesity: a simple equation with complex variables. Journal of Long-Term Effects of Medical Implants 200515 15-32.

2 Popkin BM. The nutrition transition: an overview of world patterns of change. Nutrition Reviews 200462 S140-S143.

3 Schönau E. The peak bone mass concept: is it still relevant? Pediatric Nephrology 200419 828-831.

4 Parfitt AM. The two faces of growth: benefits and risks to bone integrity. Osteoporosis International 19944 382-398.

5 Rauch F \& Schoenau E. The developing bone: slave or master of its cells and molecules? Pediatric Research 200150 309-314.

6 Frost HM. Bone 'mass' and the 'mechanostat': a proposal. Anatomical Record 1987219 1-9.

7 Burr DB. Muscle strength, bone mass, and age-related bone loss. Journal of Bone and Mineral Research 199712 1547-1551.

8 Schoenau E \& Frost HM. The 'muscle-bone unit' in children and adolescents. Calcified Tissue International 2002 70 405-407.

9 Turner CH. Muscle-bone interactions, revisited. Bone 200027 339-340.

10 Frost HM. On our age-related bone loss: insights from a new paradigm. Journal of Bone and Mineral Research 1997 12 1539-1546.

11 Frost HM \& Schonau E. The 'muscle-bone unit' in children and adolescents: a 2000 overview. Journal of Pediatric Endocrinology and Metabolism 200013 571-590.

12 Cointry GR, Capozza RF, Negri AL, Roldán EJ \& Ferretti JL Biomechanical background for a noninvasive assessment of bone strength and muscle-bone interactions. Journal of Musculoskeletal and Neuronal Interactions 20044 1-11.
13 Neu CM, Manz F, Rauch F \& Schoenau E. Bone densities and bone size at the distal radius in healthy children and adolescents: a study using peripheral quantitative computed tomography. Bone $200128227-232$.

14 Neu CM, Manz F, Rauch F \& Schoenau E. Modeling of crosssectional bone size, mass and geometry at the proximal radius: a study of normal bone development using peripheral quantitative computed tomography. Osteoporosis International 20019 $538-547$.

15 Schoenau E, Neu CM, Rauch F \& Manz F. The development of bone strength at the proximal radius during childhood and adolescence. Journal of Clinical Endocrinology and Metabolism 200186 613-618.

16 Schoenau E. From mechanostat theory to development of the 'functional muscle-bone unit'. Journal of Musculoskeletal and Neuronal Interactions $2005 \mathbf{5} 232-238$.

17 Schoenau E, Neu CM, Beck B, Manz F \& Rauch F. Bone mineral content per muscle cross-sectional area as an index of the functional muscle-bone unit. Journal of Bone and Mineral Research 200217 1095-1101.

18 Schoenau E. The functional muscle-bone-unit: a two-step diagnostic algorithm in pediatric bone disease. Pediatric Nephrology $200520356-359$.

19 Schoenau E, Neu CM, Mokov E, Wassmer G \& Manz F. Influence of puberty on muscle area and cortical bone area of the forearm in boys and girls. Journal of Clinical Endocrinology and Metabolism 200085 1095-1098.

20 Rauch F, Neu CM, Wassmer G, Beck B, Rieger-Wettengl G, Rietschel E, Manz F \& Schoenau E. Muscle analysis by measurement of maximal isometric grip force: new reference data and clinical applications in pediatrics. Pediatric Research $200251505-510$.

21 Schoenau E, Fricke O \& Rauch F. The regulation of bone development as a biological system. Homo 200354 113-118.

22 Rauch F, Travers R \& Glorieux FH. Pamidronate in children with osteogenesis imperfecta: histomorphometric effects of longterm therapy. Journal of Clinical Endocrinology and Metabolism 200691 511-516.

23 Fricke O \& Schoenau E. Influence of mechanical signaling on bone development in children and adolescents. Current Opinion in Orthopaedics 200617 443-450.

24 Julius Wolff (1836-1902) Morphogenesis of bone. Journal of the American Medical Association 19702132260.

25 Fricke O, Weidler J, Tutlewski B \& Schoenau E. Mechanography - a new device for the assessment of muscle function in pediatrics. Pediatric Research $20065946-49$.

Received 5 September 2008

Accepted 6 September 2008 